\title{
Elementary Results on Solutions to the Bellman Equation of Dynamic Programming: Existence, Uniqueness, and Convergence*
}

\author{
Takashi Kamihigashi $^{\dagger}$
}

October 15, 2013

\begin{abstract}
We establish some elementary results on solutions to the Bellman equation without introducing any topological assumption. Under a small number of conditions, we show that the Bellman equation has a unique solution in a certain set, that this solution is the value function, and that the value function can be computed by value iteration with an appropriate initial condition. In addition, we show that the value function can be computed by the same procedure under alternative conditions. We apply our results to two optimal growth models: one with a discontinuous production function and the other with "roughly increasing" returns.
\end{abstract}

Keywords: Dynamic programming, Bellman equation, value function, fixed point.

JEL Classification: C61

${ }^{*}$ I would like to thank participants at the 11th SAET Conferance in Faro, 2011, the Workshop in Honor of Cuong Le Van in Exeter, 2011, a seminar at the Paris School of Economics in 2012, and the 21st European Workshop on General Equilibrium Theory in Exeter, 2012 - especially, Larry Blume, Myrna Wooders, Felix Kübler, Juan Pablo Rincón-Zapatero, V. Filipe Martins-da-Rocha, Yiannis Vailakis, Cuong Le Van, Kevin Reffett - for helpful comments and discussions. This paper has benefited from comments and suggestions by three anonymous referees and an associate editor. Financial support from the Japan Society for the Promotion of Science is gratefully acknowledged.

${ }^{\dagger}$ RIEB, Kobe University, Rokkodai, Nada, Kobe 657-8501 JAPAN. Email: tkamihig@rieb.kobe-u.ac.jp. Tel/Fax: +81-78-803-7015. 


\section{Introduction}

Dynamic programming has been one of the most fundamental tools in economic analysis, particularly since Stokey and Lucas (1989).1 One of the limitations of Stokey and Lucas' work and earlier studies was that models with unbounded returns were not fully addressed though such models are extremely common in economics. This has been a major issue in the subsequent economic literature on dynamic programming, and important contributions have been made by Alvarez and Stokey (1998), Durán (2000), Le Van and Morhaim (2002), Rincón-Zapatero and Rodríguez-Palmero (2003, 2007, 2009), Martins-da-Rocha and Vailakis (2010), and Matkowski and Nowak (2011).

One thing common to the results established by these recent contributions is that they rely heavily on topological assumptions, such as the continuity of the return function and that of the feasibility correspondence. While these assumptions are completely standard in many cases, there are important cases in which they clearly fail. For example, threshold effects are associated with discontinuous technologies (Azariadiz and Drazen, 1990); moreoever, discontinuities are a common feature of ecological phenomena (Muradian, 2001; Nævdal, 2003).2 Even a well-behaved maximization problem that depends continuously on a parameter often results in discontinuities since parametric continuity of the objective function does not imply parametric continuity of the solution $3^{3}$ Thus, in general, discontinuities are widespread in principalagent models and game theoretic models (though such models are beyond the scope of this paper). ${ }^{4}$

The purpose of this paper is to establish some elementary results on solutions to the Bellman equation - or fixed points of the Bellman operatorwithout introducing any topological assumption. Such results not only apply

\footnotetext{
${ }^{1}$ Recent applications of dynamic programming include Algan et al. (2011), Bloch and Houy (2012), Dutta and Radner (2012), Goenka and Lin (2012), Karp and Zhang (2012), Llanes and Trento (2012), Roy and Zilcha (2012), Herrera and Martinelli (2013), Reis (2013), Schaar et al. (2013).

${ }^{2}$ See Kamihigashi and Roy $(2006,2007)$ for optimal growth models with discontinuous technologies.

${ }^{3}$ Recall that the Theorem of the Maximum (e.g., Stokey and Lucas, 1989, Theorem 3.6) shows that parametric continuity of the objective function only implies upper hemicontinuity of the solution correspondence, which means that the correspondence is in general not continuous. See Dutta and Mitra (1989a, 1989b) for related discussions.

${ }^{4}$ See Kamihigashi and Furusawa (2010, Figure 5) for a simple example.
} 
to models with discontinuities but also disentangle basic properties of fixed points of the Bellman operator from topological assumptions. One might think that in our approach, topological assumptions would simply be replaced by alternative, more abstract assumptions, but that is certainly not the case. In fact the conditions required for each formal result proved in this paper are all included in the statement of the result itself, and they are expressed mostly in terms of inequalities between functions. Therefore, our results are highly accessible to applied researchers as well as graduate students.

Our main results are twofold. First, given an order interval of functions that is mapped into itself by the Bellman operator, if the upper and lower boundaries of this order interval satisfy transversality-like conditions, then (a) the Bellman operator has a unique fixed point in the order interval; (b) this fixed point is the value function; and (c) the value function can be computed by value iteration starting from the lower boundary of the order interval. Second, if there exists a function that is mapped upward by the Bellman operator, and if this function is dominated by the value function and satisfies a transversality-like condition, then the value function can be computed by value iteration starting from this function.

To understand why such results can be shown without any topological assumption, note that most of the recent contributions except for Le Van and Morhaim (2002) are based on variants of the contraction mapping theorem. In this approach, the Bellman operator is shown to be some type of contraction, and a variant of the contraction mapping theorem is used to show that the Bellman operator has a unique fixed point in the space of continuous functions with a suitable topology. Then this fixed point is shown to be the value function using standard arguments.

However, the technical conditions required for these results imply that any fixed point in the function space under consideration must be the value function. Since there is only one value function, it follows that the uniqueness of a fixed point can be guaranteed without using any variant of the contraction mapping theorem. The idea of our approach is to use the Knaster-Tarski fixed point theorem instead of a variant of the contraction mapping theorem, and to impose a minimal set of assumptions required for the application of the Knaster-Tarski theorem. It turns out that this approach requires no topological assumption, yielding results that are considerably simpler than most existing results. We clarify this point by comparing one of our main results with the recent result developed by Rincón-Zapatero and Rodríguez-Palmero (2003, 2009) and Martins-da-Rocha and Vailakis (2010). 
There is of course a downside to our approach, which offers only a minimal convergence result and directly establishes no property of the value function except that it lies in the given order interval. On the other hand, in most cases, our convergence result is sufficient for computing the value function, and it is also useful in establishing certain properties of the value function under additional conditions. We discuss the latter point in some detail in Subsection 3.2.

In some sense, this paper is intended to be a continuation of Section 4.1 of Stokey and Lucas (1989), in which several fundamental results on dynamic programming were developed without topological assumptions. Stokey and Lucas introduced topological assumptions in their subsequent analysis, but it is possible to continue further without introducing any topological assumption. Our results are also similar in spirit to those shown by Bertsekas and Shreve (1978, Chapter 5), who mainly used monotonicity arguments. Our results extend some of their results to cases in which the return function is unbounded both above and below. In Sections 2 and 3 we offer detailed discussions of how our results relate to existing ones, including the relevant results of Stokey and Lucas (1989) and Bertsekas and Shreve (1978).

The rest of the paper is organized as follows. In the next section, we describe our framework and state our main results, which we prove in the Appendix. In Section 3, we demonstrate how they can be used to derive other useful results, and discuss related results in the literature. In Section 4, we present three examples. The first two examples are optimal growth models with logarithmic utility: one having a discontinuous production function and the other having "roughly increasing" returns. The third example demonstrates that value iteration may fail to converge to the value function unless the initial function is chosen appropriately. In Section 5 we conclude the paper.

\section{Main Results}

Let $X$ be a set, and $\Gamma$ be a nonempty-valued correspondence from $X$ to $X$. Let $D$ be the graph of $\Gamma$ :

$$
D=\{(x, y) \in X \times X: y \in \Gamma(x)\} .
$$

Let $u: D \rightarrow[-\infty, \infty)$. In the optimization problem introduced below, $X$ is the state space, $\Gamma$ is the feasibility correspondence, $u$ is the return function, 
and $D$ is the domain of $u$.

Let $\Pi$ and $\Pi\left(x_{0}\right)$ denote the set of feasible paths and that of feasible paths from $x_{0}$, respectively:

$$
\begin{aligned}
\Pi & =\left\{\left\{x_{t}\right\}_{t=0}^{\infty} \in X^{\infty}: \forall t \in \mathbb{Z}_{+}, x_{t+1} \in \Gamma\left(x_{t}\right)\right\} . \\
\Pi\left(x_{0}\right) & =\left\{\left\{x_{t}\right\}_{t=1}^{\infty} \in X^{\infty}:\left\{x_{t}\right\}_{t=0}^{\infty} \in \Pi\right\}, \quad x_{0} \in X .
\end{aligned}
$$

Let $\beta \geq 0$. Given $x_{0} \in X$, consider the following optimization problem:

$$
\sup _{\left\{x_{t}\right\}_{t=1}^{\infty} \in \Pi\left(x_{0}\right)} \underset{T \uparrow \infty}{\mathrm{L}} \sum_{t=0}^{T} \beta^{t} u\left(x_{t}, x_{t+1}\right),
$$

where $\mathrm{L} \in\{\underline{\lim }, \overline{\lim }\}$ with $\underline{\lim }=\lim \inf$ and $\varlimsup \lim =\lim$ sup. Since $u(x, y)<\infty$ for all $(x, y) \in D$, the objective function is well defined for any feasible path.

For $\left\{x_{t}\right\}_{t=0}^{\infty} \in \Pi$, we define

$$
S\left(\left\{x_{t}\right\}_{t=0}^{\infty}\right)=\underset{T \uparrow \infty}{\mathrm{L}} \sum_{t=0}^{\infty} \beta^{t} u\left(x_{t}, x_{t+1}\right) .
$$

The value function $v^{*}: X \rightarrow \overline{\mathbb{R}}$ is defined by

$$
v^{*}\left(x_{0}\right)=\sup _{\left\{x_{t}\right\}_{t=1}^{\infty} \in \Pi\left(x_{0}\right)} S\left(\left\{x_{t}\right\}_{t=0}^{\infty}\right), \quad x_{0} \in X .
$$

Note that $v^{*}\left(x_{0}\right)$ is unaffected if $\Pi\left(x_{0}\right)$ is replaced by $\Pi^{0}\left(x_{0}\right) \cdot 5^{5}$ where

$$
\begin{aligned}
\Pi^{0} & =\left\{\left\{x_{t}\right\}_{t=0}^{\infty} \in \Pi: S\left(\left\{x_{t}\right\}_{t=0}^{\infty}\right)>-\infty\right\}, \\
\Pi^{0}\left(x_{0}\right) & =\left\{\left\{x_{t}\right\}_{t=1}^{\infty} \in \Pi\left(x_{0}\right):\left\{x_{t}\right\}_{t=0}^{\infty} \in \Pi^{0}\right\}, \quad x_{0} \in X .
\end{aligned}
$$

Let $V$ be the set of functions from $X$ to $[-\infty, \infty)$. The Bellman operator $B$ on $V$ is defined by

$$
(B v)(x)=\sup _{y \in \Gamma(x)}\{u(x, y)+\beta v(y)\}, \quad x \in X, v \in V .
$$

Given $v \in V$, it need not be the case that $B v \in V$. A fixed point of $B$ is a function $v \in V$ such that $B v=v$. It follows from Kamihigashi (2008, Theorem 2) that $v^{*}$ is a fixed point of $B$ provided that $v^{*} \in V$ :

\footnotetext{
${ }^{5}$ We follow the convention that $\sup \emptyset=-\infty$.
} 
Lemma 2.1. If $v^{*} \in V$, then $v^{*}$ is a fixed point of $B$.

This is essentially the same result as Theorem 4.2 of Stokey and Lucas (1989). The difference is that Lemma 2.1 does not assume that $u(x, y)>-\infty$ for all $(x, y) \in D$; this assumption is often violated in applications. Instead the lemma assumes that $v^{*} \in V$.

Let $v, w: X \rightarrow \overline{\mathbb{R}}$. The partial order $\leq$ on the set of functions from $X$ to $\overline{\mathbb{R}}$ is defined in the usual way:

$$
v \leq w \quad \Longleftrightarrow \quad \forall x \in X, v(x) \leq w(x)
$$

The partial order $\leq$ on the set of functions from $D$ to $\overline{\mathbb{R}}$ is defined in the same way. It is immediate from $(2.9)$ that $B$ is a monotone operator on $V$ :

$$
v \leq w \in V \quad \Rightarrow \quad B v \leq B w .
$$

If $v \leq w$, we define the order interval $[v, w]$ by

$$
[v, w]=\{f \in V: v \leq f \leq w\}
$$

The order interval $[-\infty, w]$ means $[v, w]$ with $v=-\infty$; likewise $[v, \infty]$ means $[v, w]$ with $w=\infty .6$

Now we are ready to state the main results of this paper, the proofs of which appear in the Appendix.

Theorem 2.1. Suppose that there exist $\underline{v}, \bar{v} \in V$ such that

$$
\begin{gathered}
\underline{v} \leq \bar{v}, \\
B \underline{v} \geq \underline{v}, \\
B \bar{v} \leq \bar{v}, \\
\forall\left\{x_{t}\right\}_{t=0}^{\infty} \in \Pi^{0}, \quad \underline{\lim }_{t \uparrow \infty} \beta^{t} \underline{v}\left(x_{t}\right) \geq 0, \\
\forall\left\{x_{t}\right\}_{t=0}^{\infty} \in \Pi, \quad \varlimsup_{t \uparrow \infty} \beta^{t} \bar{v}\left(x_{t}\right) \leq 0 .
\end{gathered}
$$

Then the following conclusions hold:

(a) The Bellman operator $B$ has a unique fixed point in $[\underline{v}, \bar{v}]$.

(b) This fixed point is the value function $v^{*}$.

${ }^{6}$ Given $r \in \overline{\mathbb{R}}$, the notation $r$ is understood as the extended-real number $r$ or the function identically equal to $r$ depending on the context. 
(c) The increasing sequence $\left\{B^{n} \underline{v}\right\}_{n=1}^{\infty}$ converges to $v^{*}$ pointwise. $]^{7}$

In conclusion (c) above, the sequence $\left\{B^{n} \underline{v}\right\}_{n=1}^{\infty}$ is increasing because we have $B^{n+1} \underline{v} \geq B^{n} \underline{v}$ for any $n \in \mathbb{N}$ by applying $B^{n}$ to both sides of (2.14). The next two results follow from intermediate steps in the the proof of Theorem 2.1 .

Proposition 2.1. Suppose that there exists $\bar{v} \in V$ satisfying (2.17) and

$$
v^{*} \leq \bar{v}
$$

Then $v^{*}$ is the largest fixed point of $B$ in $[-\infty, \bar{v}] !^{8}$

Theorem 2.2. Suppose that $v^{*} \in V$. Suppose further that there exists $\underline{v} \in V$ satisfying (2.14), (2.16), and

$$
\underline{v} \leq v^{*} .
$$

Then $v^{*}$ is the smallest fixed point of $B$ in $[\underline{v}, \infty]$. Furthermore, the increasing sequence $\left\{B^{n} \underline{v}\right\}_{n=1}^{\infty}$ converges to $v^{*}$ pointwise.

Proposition 2.1 and the first conclusion of Theorem 2.2 can be shown by extending the proof of Stokey and Lucas (1989, Theorem 4.3) to our slightly more general setting (though we do not entirely follow this route). Their argument, which assumes the stronger version of 2.16 with $\Pi$ in place of $\Pi^{0}$, in fact goes through even with the original version of (2.16). The idea of replacing $\Pi$ with $\Pi^{0}$ is due to Le Van and Morhaim (2002). This replacement is important because even the value function $v^{*}$ almost never satisfies the stronger version of (2.16) if the return function is unbounded below. See Subsection 3.3 for related conditions used by Rincón-Zapatero and Rodríguez-Palmero (2003) and Martins-da-Rocha and Vailakis (2010) ${ }^{9}$

To our knowledge, the second conclusion of Theorem 2.2 is new. If it is known that $v^{*} \in[\underline{v}, \bar{v}]$, which implies both (2.18) and (2.19), then Theorem 2.1 follows directly from Proposition 2.1 and Theorem 2.2 But since it is

\footnotetext{
${ }^{7}$ In this paper, "increasing" means "nondecreasing," "decreasing" means "nonincreasing," "positive" means "nonnegative," and "negative" means "nonpositive."

${ }^{8}$ This means that any fixed point $v$ of $B$ in $[-\infty, \bar{v}]$ satisfies $v \leq v^{*}$. A similar remark applies to the first conclusion of Theorem 2.2 .

${ }^{9}$ Rincón-Zapatero and Rodríguez-Palmero (2003, Theorem 5) also use a function satisfying (2.17) as an upper bound on an increasing sequence of fixed points of the Bellman operators corresponding to truncated problems.
} 
not known a priori whether $v^{*}$ lies in $[\underline{v}, \bar{v}]$, the additional contribution of Theorem 2.1 is to show that $v^{*}$ indeed lies in $[\underline{v}, \bar{v}]$.

If there exist functions $\underline{v}, \bar{v} \in V$ satisfying (2.13)-(2.15), then the Bellman operator $B$ has a fixed point in $[\underline{v}, \bar{v}]$ by the Knaster-Tarski fixed point theorem (see the Appendix for a precise argument). However, this fixed point need not be the value function $v^{*}$. In fact, under 2.13)-(2.15), we do not even know whether $v^{*}$ lies in $V$. Even if it does, it still need not lie in $[\underline{v}, \bar{v}]$ though it is a fixed point of $B$ by Lemma 2.1.

Therefore, additional conditions are needed to show that $v^{*} \in[\underline{v}, \bar{v}]$. Conditions (2.16) and (2.17), which we called transversality-like conditions in the Introduction, serve this purpose, implying that any fixed point of $B$ in $[\underline{v}, \bar{v}]$ must be the value function $v^{*}$. Since we already know that $B$ has a fixed point in $[\underline{v}, \bar{v}]$, this fixed point must be the value function $v^{*}$, so that $v^{*}$ lies in $[\underline{v}, \bar{v}]$. The uniqueness of a fixed point of $B$ in $[\underline{v}, \bar{v}]$ follows from the fact that any fixed point in $[\underline{v}, \bar{v}]$ equals $v^{*}$. Rincón-Zapatero and RodríguezPalmero (2003) offer several nontrivial, economically relevant examples satisfying stronger versions of (2.13)-(2.17).

If (2.16) and (2.17) are violated, then the Bellman operator $B$ can have multiple fixed points in $[\underline{v}, \bar{v}]$; see Kamihigashi (2013). It follows that conditions such as (2.16) and (2.17) are crucial to ensuring that $v^{*}$ is the only fixed point of $B$ in $[\underline{v}, \bar{v}]$.

Note that in conclusion (c) of Theorem 2.1, convergence to $v^{*}$ is guaranteed only from $\underline{v}$. Our argument for this convergence result is based on the simple observation that the limit of the increasing sequence $\left\{B^{n} \underline{v}\right\}_{n=1}^{\infty}$ is the supremum of the sequence. This allows us to interchange this supremum and another supremum (see (A.16) A.18) to show that the limit is the value function $v^{*}$. The case of the decreasing sequence $\left\{B^{n} \bar{v}\right\}_{n=1}^{\infty}$, which also converges pointwise, is not symmetric since the sup and inf operators are in general not interchangeable. In Subsection 4.3 we construct an example satisfying 2.13)-2.17) in which $\lim _{n \uparrow \infty} B^{n} \bar{v} \neq v^{*}{ }^{10}$

Recall that the definition of the value function $v^{*}$ in $(2.6)$ depends on the definition of $\mathrm{L}$, which can be $\underline{\lim }$ or $\overline{\lim }$. Thus there are in fact two value functions: one with $\mathrm{L}=\underline{\lim }$ and the other with $\mathrm{L}=\varlimsup$ lim. An interesting implication of Theorem 2.1 is that these two functions coincide under (2.13)(2.17). This is because the unique fixed point of $B$ established in Theorem 2.1 depends only on $B$, which is independent of $\mathrm{L}$.

\footnotetext{
${ }^{10}$ See Strauch (1966, p. 880) for a related example of an undiscounted stochastic model.
} 


\section{Corollaries}

\subsection{Special Cases}

In this subsection we show various special cases of the results shown in the previous section to illustrate how our results can be used to derive other useful results. Many of the results shown here are more or less known, but these results are intended to clarify how our results relate to existing ones. The last two results in this subsection are new and useful in our view. Let us start with a simple consequence of Proposition 2.1.

Corollary 3.1. Suppose that there exists $\bar{v} \in V$ satisfying (2.15), (2.17), and (2.18). Let $\bar{v}^{*}$ be the pointwise limit of the decreasing sequence $\left\{B^{n} \bar{v}\right\}_{n=1}^{\infty}$. Then $v^{*} \leq \bar{v}^{*}$. Furthermore, if $\bar{v}^{*}$ is a fixed point of $B$, then $\bar{v}^{*}=v^{*}$.

Proof. The inequality $v^{*} \leq \bar{v}^{*}$ is immediate from 2.15) and (2.18). If $\bar{v}^{*}$ is a fixed point of $B$, then $\bar{v}^{*} \leq v^{*}$ by Proposition 2.1; thus $\bar{v}^{*}=v^{*}$.

The above result is essentially equivalent to Theorem 4.14 of Stokey and Lucas (1989). Corollary 3.1 along with Proposition 2.1 implies the following.

Corollary 3.2. Suppose that $u \leq 0$. Then $v^{*}$ is the largest negative fixed point of $B$. Let $\bar{v}^{*}$ be the pointwise limit of the decreasing sequence $\left\{B^{n} 0\right\}_{n=1}^{\infty}$. Then $v^{*} \leq \bar{v}^{*}$. Furthermore, if $\bar{v}^{*}$ is a fixed point of $B$, then $\bar{v}^{*}=v^{*}$.

Proof. Since $u \leq 0$, we have $B 0 \leq 0$ and $v^{*} \leq 0$; thus (2.15) and (2.18) hold with $\bar{v}=0$. In addition (2.17) trivially holds. Hence the conclusions hold by Proposition 2.1 and Corollary 3.1.

The above result is essentially equivalent to Proposition 5.8 of Bertsekas and Shreve (1978). The following result deals with the case $u \geq 0$.

Corollary 3.3. Suppose that $u \geq 0$, and that $v^{*} \in V$. Then $v^{*}$ is the smallest positive fixed point of $B$, and the increasing sequence $\left\{B^{n} 0\right\}_{n=1}^{\infty}$ converges to $v^{*}$ pointwise.

Proof. Since $u \geq 0$, we have $B 0 \geq 0$ and $v^{*} \geq 0$; thus 2.14) and (2.19) hold with $\underline{v}=0$. In addition (2.16) trivially hold. Hence the conclusions hold by Theorem 2.2 . 
The above result is essentially equivalent to Proposition 5.7 of Bertsekas and Shreve (1978). Corollaries 3.2 and 3.3 indicate that our results in the previous section extend their results to problems in which the return function is unbounded both above and below. Since such return functions are common in economics, this extension is significant. It is also nontrivial given that Bertsekas and Shreve's (1978, Chapter 5) arguments rely heavily on the assumption that the return function takes only one sign, as assumed in Corollaries 3.2 and 3.3 above.

Next we consider the case in which the return function $u$ is bounded:

Corollary 3.4. Suppose that $\beta \in[0,1)$. Suppose further that there exist constants $\underline{\mu}, \bar{\mu} \in \mathbb{R}$ such that

$$
\underline{\mu} \leq u \leq \bar{\mu}
$$

Then conclusions (a)-(c) of Theorem 2.1 hold with

$$
\underline{v}=\frac{\underline{\mu}}{1-\beta}, \quad \bar{v}=\frac{\bar{\mu}}{1-\beta} .
$$

Proof. We have $\underline{v} \leq \bar{v}$ by construction. Note that for any $x \in X$ we have

$$
\begin{aligned}
(B \underline{v})(x) & =\sup _{y \in \Gamma(x)}\{u(x, y)+\beta \underline{v}(y)\} \\
& \geq \sup _{y \in \Gamma(y)}\{\underline{\mu}+\beta \underline{\mu} /(1-\beta)\}=\underline{\mu} /(1-\beta)=\underline{v}(x) .
\end{aligned}
$$

Thus $B \underline{v} \geq \underline{v}$. We similarly obtain $B \bar{v} \leq \bar{v}$. Hence 2.13)-2.15 hold. Since $\underline{v}$ and $\bar{v}$ are constant functions, (2.16) and (2.17) trivially hold. Conclusions (a)-(c) of Theorem 2.1 now follow.

The above result is similar to the well known result that if $u$ is bounded and continuous, and if $\Gamma$ is continuous and compact-valued, then the Bellman operator has a unique fixed point in the space of bounded continuous functions, this fixed point is the value function, and value iteration converges uniformly to the value function starting from any bounded continuous function (Stokey and Lucas, 1989, pp. 79-80). Corollary 3.4 shows that similar conclusions can be obtained without any topological assumption except for the globally uniform convergence result. Corollary 3.4 is particularly useful to applied researchers and graduate students because it can be shown and 
used without knowing mathematical concepts such as metric spaces, contractions, and completeness. In addition, even under standard topological assumptions, the approach based on the contraction mapping theorem fails as soon as one inequality is removed from (3.1), but our approach continues to work:

Corollary 3.5. Suppose that $\beta \in[0,1)$, and that $v^{*} \in V$. Suppose further that there exists a constant $\mu \in \mathbb{R}$ such that $u \geq \mu$. Then $v^{*}$ is the smallest fixed point $v$ of $B$ with $v \geq \underline{\mu} /(1-\beta)$, and the increasing sequence $\left\{B^{n} \underline{\mu}\right\}_{n=1}^{\infty}$ converges to $v^{*}$ pointwise.

Proof. Let $\underline{v}=\mu /(1-\beta) \in V$. Then $(2.14)$ and $(2.16)$ hold as in the proof of Corollary 3.4. In addition 2.19 is immediate from the inequality $u \geq \underline{\mu}$. Thus the conclusions hold by Theorem 2.2 .

The above result is similar to Corollary 3.3 , but requires the additional assumption that $\beta \in[0,1)$. To our knowledge, the next two results are new although they are similar to Corollaries 3.4 and 3.5. The new results show how one can construct functions $\underline{v}$ and $\bar{v}$ that can be used to apply Theorems 2.1 and/or 2.2 even when the conditions of Corollaries 3.4 and 3.5 are too restrictive; see Section 4.1 for applications.

Corollary 3.6. Suppose that there exist functions $\underline{u}, \bar{u}: D \rightarrow[-\infty, \infty)$ such that

$$
\underline{u} \leq u \leq \bar{u} .
$$

Suppose further that there exist correspondences $\underline{\Gamma}, \bar{\Gamma}: X \rightarrow X$ such that

$$
\forall x \in X, \quad \underline{\Gamma}(x) \subset \Gamma(x) \subset \bar{\Gamma}(x) .
$$

Let $\underline{v}: X \rightarrow \overline{\mathbb{R}}$ be the value function given by (2.6) with $\underline{u}$ and $\underline{\Gamma}$ replacing $u$ and $\Gamma$, respectively. Let $\bar{v}: X \rightarrow \overline{\mathbb{R}}$ be the value function given by (2.6) with $\bar{u}$ and $\bar{\Gamma}$ replacing $u$ and $\Gamma$, repectively. Suppose that $\bar{v} \in V$, and that 2.16) and (2.17) hold. Then conclusions (a)-(c) of Theorem 2.1 hold.

Proof. We have $\underline{v} \leq \bar{v}$ by construction. Since $\underline{v} \leq \bar{v} \in V$, we have $\underline{v} \in V$ as well. Note that for any $x \in X$ we have

$$
\begin{aligned}
(B \underline{v})(x) & =\sup _{y \in \Gamma(x)}\{u(x, y)+\beta \underline{v}(y)\} \\
& \geq \sup _{y \in \underline{\Gamma}(y)}\{\underline{u}(x, y)+\beta \underline{v}(y)\}=\underline{v}(x),
\end{aligned}
$$


where the last equality holds by Lemma 2.1. It follows that $B \underline{v} \geq \underline{v}$. We similarly obtain $B \bar{v} \leq \bar{v}$. Now (2.13)-2.15) hold. Since (2.16) and (2.17) hold by assumption, conclusions (a)-(c) of Theorem 2.1 now follow.

Corollary 3.7. Suppose that $v^{*} \in V$, and that there exists a function $\underline{u}$ : $D \rightarrow[-\infty, \infty)$ such that $\underline{u} \leq u$. Suppose further that there exists a correspondence $\underline{\Gamma}: X \rightarrow X$ such that $\underline{\Gamma}(x) \subset \Gamma(x)$ for all $x \in X$. Define $\underline{v}$ as in Corollary 3.6. Suppose that (2.16) holds. Then $v^{*}$ is the smallest fixed point $v$ of $B$ in $[\underline{v}, \infty]$, and the increasing sequence $\left\{B^{n} \underline{v}\right\}_{n=1}^{\infty}$ converges to $v^{*}$ pointwise.

Proof. We have 2.14 and 2.16) as in the proof of Corollary 3.6. In addition (2.19) is immediate from the inequality $\underline{u} \leq u$. By (2.19), we have $\underline{v} \in V$. Thus the conclusions hold by Theorem 2.2 .

\subsection{Properties of the Value Function}

There are two obvious limitations of the convergence result in Theorems 2.1 and 2.2. First, convergence to the value function $v^{*}$ is guaranteed only from one particular function $\underline{v}$. Second, convergence occurs only pointwise. Despite these limitations, there are various situations in which this convergence result can help establish a property of the value function. This is because pointwise convergence preserves many important properties such as monotonicity, concavity, convexity, measurability, and supermodularity. In fact any property that can be expressed in terms of weak inequalities is preserved under pointwise convergence. In addition, since our convergence result deals only with a particular increasing sequence starting from $\underline{v}$, it suffices to verify that a property of interest is preserved at the limit of this sequence. The following result formalizes this idea.

Corollary 3.8. Suppose that $v^{*} \in V$. Let $\underline{v} \in V$ satisfy (2.14), (2.16), and (2.19). Let $W \subset V$ satisfy the following conditions:

(i) $\underline{v} \in W$.

(ii) For any $v \in W$ with $B v \geq v$, we have $B v \in W$.

(iii) For any increasing sequence $\left\{v_{n}\right\}_{n=1}^{\infty} \subset W$ with $\lim _{n \uparrow \infty} v_{n} \in V$, we have $\lim _{n \uparrow \infty} v_{n} \in W$.

Then $v^{*} \in W$. 
Proof. Let $\underline{v}$ and $W$ be as above. For $n \in \mathbb{N}$, let $\underline{v}_{n}=B^{n} \underline{v}$, and let $\underline{v}^{*}=$ $\lim _{n \uparrow \infty} \underline{v}_{n}$. By Theorem 2.2, we have $\underline{v}^{*}=v^{*}$. Since $\underline{v} \in W$ by condition (i), and since the sequence $\left\{B^{n} \underline{v}\right\}$ is increasing by (2.14), we have $\underline{v}_{n} \in W$ for all $n \in \mathbb{N}$ by condition (ii). By condition (iii) we have $\underline{v}^{*} \in W$; that is, $v^{*} \in W$.

To see how the above result can be used in practice, suppose that one wishes to establish that the value function has a certain property, say property $(\mathrm{P})$. One can do so in four steps as follows:

I. Find a function $\underline{v} \in V$ with property $(\mathrm{P})$ that satisfies (2.14), (2.16), and (2.19).

II. Show that if a function $v \in V$ has property $(\mathrm{P})$ and satisfies $B v \geq v$, then $B v$ also has property $(\mathrm{P})$.

III. Show that for any increasing sequence $\left\{v_{n}\right\}$ such that each $v_{n}$ has property $(\mathrm{P}), \lim _{n \uparrow \infty} v_{n}$ has property $(\mathrm{P})$.

IV. Using Corollary 3.8, conclude that the value function $v^{*}$ has property $(\mathrm{P})$.

This type of argument is not new (e.g., Stokey and Lucas, 1989, p. 52; Amir et al., 1991, p. 637), but note that it is the convergence result in Theorems 2.1 and 2.2 that makes the above procedure work. In Subsection 4.2 we apply a slightly different version of this procedure to an optimal growth model with "roughly increasing" returns.

\subsection{Comparison with the Result Shown by Rincón- Zapatero and Rodríguez-Palmero/Martins-da-Rocha and Vailakis}

We now compare Theorem 2.1 with Martins-da-Rocha and Vailakis' (2010, Sec. 3) result on the Bellman operator, which is built upon the work of Rincón-Zapatero and Rodríguez-Palmero (2003, 2009). Under numerous technical conditions, their result shows that the Bellman operator has a unique fixed point in a certain set of continuous functions, that this fixed point is the value function, and that value iteration converges locally uniformly to the value function starting from any continuous function in the given function set. Since our result does not establish any property of the value function and ensures only pointwise convergence from a specific initial 
function, the conclusions of our result are weaker in some directions. However, our result, which requires only a small subset of the conditions required by their result, is considerably simpler to apply. This point immediately becomes clear in what follows.

The result stated by Martins-da-Rocha and Vailakis (2010, Theorem 3.1) requires the assumptions listed below:

DP0. (i) $X=\mathbb{R}_{+}^{m}$ for some $m \in \mathbb{N}$. (ii) $\beta \in(0,1)$. (iii) The right-hand side of 2.5 with $\mathrm{L}=\mathrm{lim}$ exists in $\overline{\mathbb{R}}$ for all $\left\{x_{t}\right\}_{t=0}^{\infty} \in \Pi$.

DP1. The feasibility correspondence $\Gamma$ is continuous and compact-valued.

DP2. The return function $u$ is continuous on $D$.

Let $C(X,[-\infty, \infty))$ be the set of continuous functions from $X$ to $[-\infty, \infty)$. Under DP0(i), we define

$$
\begin{aligned}
X^{*} & =X \backslash\{0\}, \\
C^{*}(X) & =\left\{v \in C(X,[-\infty, \infty)): \forall x \in X^{*}, v(x)>-\infty\right\} .
\end{aligned}
$$

Though DP2 requires $u$ to be continuous, it is allowed that $u(x, y)=-\infty$ for some $(x, y) \in D$. The following assumption requires that one can avoid $u(x, y)=-\infty$ unless $x=0$.

DP3. There exists a continuous function $q: X^{*} \rightarrow X^{*}$ such that

$$
\forall x \in X^{*}, \quad(x, q(x)) \in D, \quad u(x, q(x))>-\infty .
$$

DP4. (i) There exist $\underline{v}, \bar{v} \in C^{*}(X)$ satisfying (2.13)-2.15). (ii) We have

$$
\begin{array}{ll}
\forall\left\{x_{t}\right\}_{t=0}^{\infty} \in \Pi^{0}, & \lim _{t \uparrow \infty} \beta^{t} \underline{v}\left(x_{t}\right)=0, \\
\forall\left\{x_{t}\right\}_{t=0}^{\infty} \in \Pi^{0}, & \lim _{t \uparrow \infty} \beta^{t} \bar{v}\left(x_{t}\right)=0 .
\end{array}
$$

(iii) For any $x_{0} \in X^{*}, \Pi^{0}\left(x_{0}\right) \neq \emptyset$. (iv) There exists $\hat{v} \in C^{*}(X)$ such that

$$
\begin{gathered}
\forall x \in X^{*}, \quad \bar{v}(x)<\hat{v}(x), \quad(B \hat{v})(x)<\hat{v}(x), \\
\exists \epsilon>0, \quad \sup _{x \in X^{*}:\|x\|<\epsilon} \frac{\underline{v}(x)-\hat{v}(x)}{\bar{v}(x)-\hat{v}(x)}<\infty, \\
\exists \delta>0, \quad \sup _{x \in X^{*}:\|x\|<\delta} \frac{\bar{v}(x)-\hat{v}(x)}{(B \hat{v})(x)-\hat{v}(x)}<\infty,
\end{gathered}
$$

where $\|\cdot\|$ is any norm on $\mathbb{R}^{m}$. 
DP5. There exists an increasing sequence $\left\{K_{j}\right\}_{j=1}^{\infty}$ of compact subsets of $X$ such that $\Gamma\left(K_{j}\right) \subset K_{j}$ for each $j \in \mathbb{N}$, and such that for any compact set $K \subset X$, there exists $j \in \mathbb{N}$ with $K \subset K_{j}$.

We are now ready to present the result stated by Martins-da-Rocha and Vailakis (2010):

Theorem 3.1. (Martins-da-Rocha and Vailakis, 2010, Theorem 3.1) Under DP0-DP5, the following conclusions hold:

(a) The Bellman operator $B$ has a unique fixed point in $[\underline{v}, \bar{v}] \cap C^{*}(X)$.

(b) The unique fixed point of $B$ in $[\underline{v}, \bar{v}] \cap C^{*}(X)$ is the value function $v^{*}$.

(c) For any $v \in[\underline{v}, \bar{v}] \cap C^{*}(X)$, the sequence $\left\{B^{n} v\right\}_{n=1}^{\infty}$ converges to $v^{*}$ in the topology generated by the family $\left\{d_{j}\right\}_{j=1}^{\infty}$ of semidistances defined for all $f, g \in[\underline{v}, \bar{v}] \cap C^{*}(X)$ by

$$
d_{j}(f, g)=\sup _{x \in K_{j} \backslash\{0\}}\left|\ln \frac{f(x)-\hat{v}(x)}{\bar{v}(x)-\hat{v}(x)}-\ln \frac{g(x)-\hat{v}(x)}{\bar{v}(x)-\hat{v}(x)}\right|, \quad j \in \mathbb{N} .
$$

Let us compare the conclusions of Theorem 2.1 with those of Theorem 3.1 under DP0-DP5 (which imply the hypotheses of Theorem 2.1 by Corollary 3.9 below). In terms of the existence of a fixed point, Theorem 2.1 is weaker than Theorem 3.1 in the sense that the latter shows that the fixed point lies in $C^{*}(X)$ (and thus the value function $v^{*}$ is continuous). In terms of uniqueness, Theorem 2.1 is stronger than Theorem 3.1, which ensures uniqueness only in $[\underline{v}, \bar{v}] \cap C^{*}(X)$. In terms of convergence, Theorem 2.1 is considerably weaker than Theorem 3.1, which shows that convergence to $v^{*}$ occurs from any initial function in $[\underline{v}, \bar{v}] \cap C^{*}(X)$ under a criterion much stronger than ours.

Let us now clarify which parts of DP0-DP5 are needed for the conclusions of Theorem 2.1. The following is a corollary of Theorem 2.1.

Corollary 3.9. Assume DPO(i), DPO(ii), DP4(i), (3.12), and DP5. Then the functions $\underline{v}$ and $\bar{v}$ given by DP4(i) satisfy (2.13)-(2.17), and thus conclusions (a)-(c) of Theorem 2.1 hold.

Proof. DP4(i) ensures (2.13)-(2.15). Condition (3.12) implies (2.16). To verify (2.17), let $\left\{x_{t}\right\}_{t=0}^{\infty} \in \Pi$. By DP5, there exists a compact set $K_{j}$ with $j \in \mathbb{N}$ such that $x_{0} \in K_{j}$ and $\Gamma\left(K_{j}\right) \subset K_{j}$. Since $\bar{v}$ is continuous by DP4(i), we have $\bar{v}\left(x_{t}\right) \leq \max _{x \in K_{j}} \bar{v}(x)<\infty$ for all $t \in \mathbb{Z}_{+}$, which together with DP0(ii) implies (2.17). 
The above result shows that a small subset of the conditions stated in DP0-DP5 is sufficient to show that the value function $v^{*}$ is the unique fixed point of the Bellman operator $B$ in $[\underline{v}, \bar{v}]$ and that convergence to $v^{*}$ occurs from $\underline{v}$. In the proof, (2.17) is shown as a consequence of DP5 rather than (3.13). The only role of DP0(i) is to keep $C^{*}(X)$ well defined, while that of DP5 is to ensure (2.17). Thus DP0(i) can be dropped entirely if we do not require $\underline{v}, \bar{v} \in C^{*}(X)$, while DP5 can be replaced by a weaker sufficient condition for (2.17). The following result can be shown by modifying the proof of Corollary 3.9 .

Corollary 3.10. Let $\beta \in[0,1)$. Suppose that there exist $\underline{v}, \bar{v} \in V$ satisfying (2.13) - (2.16). Suppose further that for any $x \in X$, there exists a set $K \subset X$ such that $\Gamma(K) \subset K$ and $\sup _{x \in K} \bar{v}(x)<\infty$. Then conclusions (a)-(c) of Theorem 2.1 hold.

\section{Examples}

Throughout this section we assume that $\beta \in(0,1)$.

\subsection{Optimal Growth with Threshold Effects}

Azariadis and Drazen (1990, p. 508) define threshold effects as "radical differences in dynamic behavior arising from local variations in social returns to scale," explaining this idea by using a discontinuous production function. In this subsection we consider a one-sector growth model with this feature. The example here is a special case of the model studied by Kamihigashi and Roy (2007).

Let $\underline{\theta}, \bar{\theta}, \hat{x}>0$ with $\underline{\theta}<\bar{\theta}$. Consider the production function $f: \mathbb{R}_{+} \rightarrow \mathbb{R}$ specified by

$$
f(x)= \begin{cases}\underline{\theta} x & \text { if } x<\hat{x}, \\ \bar{\theta} x & \text { if } x \geq \hat{x} .\end{cases}
$$

Figure 1 illustrates a production function of this form. We define

$$
\begin{aligned}
u(x, y) & =\ln [f(x)-y], \quad(x, y) \in D, \\
\Gamma(x) & =\{y \in \mathbb{R}: 0 \leq y \leq f(x)\}, \quad x \in X .
\end{aligned}
$$

This is clearly a special case of the framework described in Section 2. 


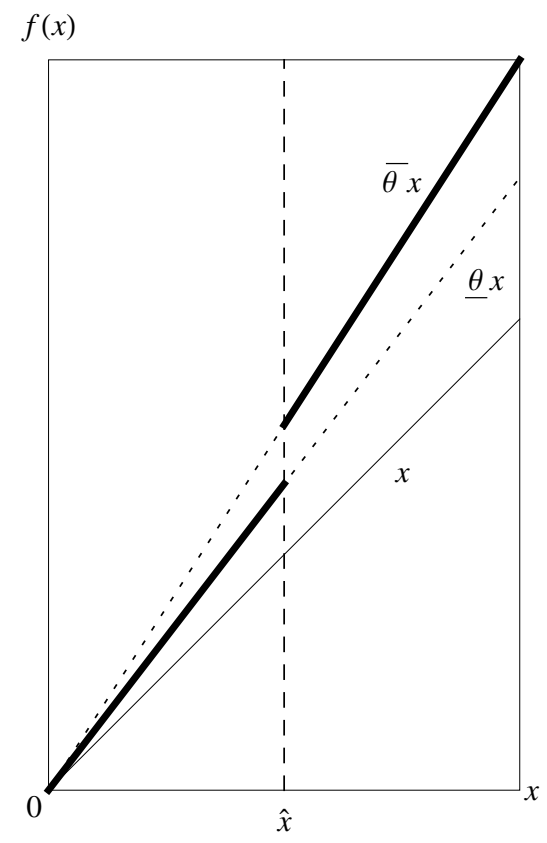

Figure 1: Production function of the form 4.1

Since the production function $f$ is discontinuous, it is immediately clear that $u$ is not a continuous function and $\Gamma$ is not a continuous correspondence ${ }^{11}$ Hence any result that requires $u$ and $\Gamma$ to be continuous is not applicable here. For example, it is easy to see that this example violates DP1 and DP2 in Subsection 3.3. It also violates DP5, which rules out unbounded growth.

In contrast, our results are easily applicable. To be specific, define

$$
\begin{gathered}
\underline{u}(x, y)=\ln (\underline{\theta} x-y), \quad \bar{u}(x, y)=\ln (\bar{\theta} x-y), \\
\underline{\Gamma}(x)=\{y \in \mathbb{R}: 0 \leq y \leq \underline{\theta} x\}, \quad \bar{\Gamma}(x)=\{y \in \mathbb{R}: 0 \leq y \leq \bar{\theta} x\} .
\end{gathered}
$$

Let $\underline{B}$ and $\bar{B}$ be the Bellman operators corresponding to $(\underline{u}, \underline{\Gamma})$ and $(\bar{u}, \bar{\Gamma})$, respectively. Then, using the method of undetermined coefficients, it is easy to show that there exist $\underline{\gamma}, \bar{\gamma}, \underline{\eta}, \bar{\eta}>0$ such that $\underline{v}=\underline{B} \underline{v}$ and $\bar{v}=\bar{B} \bar{v}$ with

$$
\underline{v}(x)=\underline{\gamma} \ln x+\underline{\eta}, \quad \bar{v}(x)=\bar{\gamma} \ln x+\bar{\eta} .
$$

\footnotetext{
${ }^{11}$ By using a different state variable, it is possible to construct a continuous return function, but the feasibility correspondence cannot be made continuous.
} 
In particular,

$$
\underline{\gamma}=\bar{\gamma}=\frac{1}{1-\beta}, \quad \underline{\eta}=\frac{1}{(1-\beta)^{2}}[\ln \underline{\theta}+\beta \ln \beta+(1-\beta) \ln (1-\beta)],
$$

and $\bar{\eta}$ is similar ${ }^{12}$ It is easy to see that $2.13-2.15$ hold. It is also easy to verify (2.16) and (2.17) following Rincón-Zapatero and Rodríguez-Palmero (2003, Example 12); thus conclusions (a)-(c) of Theorem 2.1 hold.

Note that in the above argument, it was not required to show that $\underline{v}$ and $\bar{v}$ are the value functions corresponding to $(\underline{u}, \underline{\Gamma})$ and $(\bar{u}, \bar{\Gamma})$, respectively. However, one can easily show that $\underline{v}$ and $\bar{v}$ are indeed the associated value functions by using Theorem 2.1 or Corollary 3.1.13 In that case, one can apply Corollary 3.6 or 3.7 .

In this example, the production function is assumed to have only one discontinuity and to be upper semicontinuous. However, these properties are not required by our results. Indeed, the above arguments goes through as long as the production function lies between $\underline{\theta} x$ and $\bar{\theta} x$

\subsection{Optimal Growth with Roughly Increasing Returns}

The importance of increasing returns in explaining cross-country differences in the long run has been well recognized since Romer (1986). In this subsection we consider an optimal growth model with "roughly increasing" returns, which is again a special case of the model studied by Kamihigashi and Roy (2007). In particular we assume that the production function $f$ is continuous and strictly increasing, and that there exist $\underline{\theta}, \bar{\theta} \in \mathbb{R}_{++}$and $\alpha \in(1,1 / \beta)$ such that

$$
\underline{\theta} x^{\alpha} \leq f(x) \leq \bar{\theta} x^{\alpha}
$$

Figure 2 illustrates a production function of this form, which roughly exhibits increasing returns. We define the return function $u$ and the feasibility correspondence $\Gamma$ by 4.2 .

\footnotetext{
${ }^{12}$ To derive these values of $\gamma$ and $\eta$, for example, suppose that $\underline{v}$ takes the form given by (4.6), and solve the maximization problem $\max _{y \in \Gamma(x)}\{\ln (\underline{\theta} x-y)+\beta[\underline{\gamma} \ln y+\eta]\}$. Substitute the solution into the objective function, and find the values of $\underline{\gamma}$ and $\eta \underline{\text { such that the }}$ maximized objective function always equals $\underline{\gamma} \ln x+\underline{\eta}$.

${ }^{13}$ The latter approach is used by Stokey and Lucas (1989, Section 4.4).

${ }^{14}$ Kamihigashi and Stachurski (2011) use a similar idea to analyze monotone Markov chains.
} 


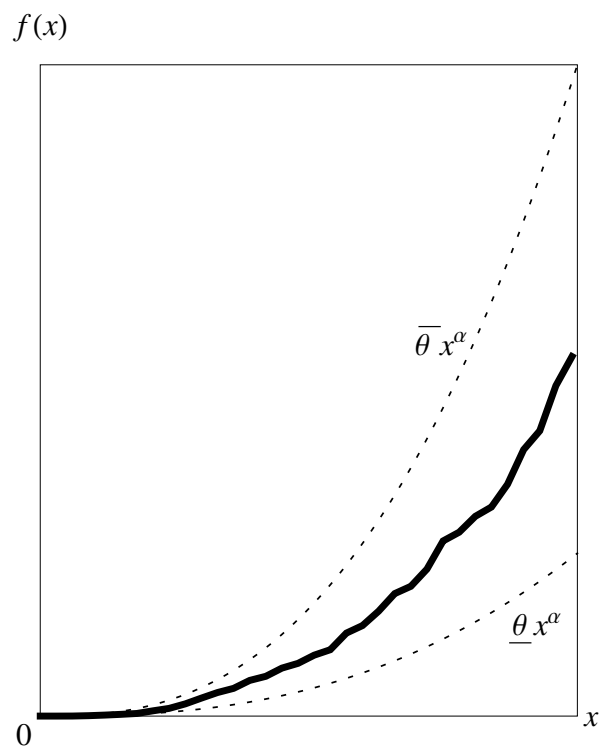

Figure 2: Production function of the form 4.8

In this example, both $u$ and $\Gamma$ are continuous, but to our knowledge, in the current literature, there is no result on the convergence of value iteration that applies to this example. However, our results are easily applicable as in the previous subsection. To demonstrate this, define $\underline{u}, \underline{\Gamma}, \bar{u}$, and $\bar{\Gamma}$ by $(4.4)$ and (4.5) with $\underline{\theta} x^{\alpha}$ and $\bar{\theta} x^{\alpha}$ replacing $\underline{\theta} x$ and $\bar{\theta} x$, respectively. Then following the procedure in the previous subsection, we see that there exist $\underline{\gamma}, \bar{\gamma}, \underline{\eta}, \bar{\eta}>0$ such that $\underline{v}=\underline{B} \underline{v}$ and $\bar{v}=\bar{B} \bar{v}$ with $\underline{v}$ and $\bar{v}$ given by 4.6); thus we can apply Theorem 2.1 in the same way. In particular, the increasing sequence $\left\{B^{n} \underline{v}\right\}$ converges to $v^{*}$ pointwise.

It follows from Kamihigashi and Roy (2007, Lemma 3.3) that the value function $v^{*}$ here is upper semicontinuous. Let us show that $v^{*}$ is in fact continuous 15 Since $\underline{v}$ is continuous, and since the Bellman operator $B$ here maps a continuous function to a continuous function, $B^{n} \underline{v}$ is continuous for each $n \in \mathbb{N}$. However, we cannot apply Corollary 3.8 here since continuity is not

\footnotetext{
${ }^{15}$ Note from Le Van and Morhaim (2002) that continuity of $v^{*}$ is not necessarily immediate. One can verify the continuity of $v^{*}$ here by following Le Van and Morhaim (2002, Theorem 3(iii)), even though their assumptions rule out increasing returns. One can also verify it more directly using the interiority of optimal paths. However, given that $v^{*}$ is known to be upper semicontinuous, the following argument seems to be quite efficient.
} 
preserved under pointwise convergence. On the other hand, the pointwise limit of an increasing sequence of continuous functions is lower semicontinuous. This suffices for our purpose since then $v^{*}$ is both upper and lower semicontinuous, i.e., $v^{*}$ is continuous.

\subsection{Nonconvergence of $\left\{B^{n} \bar{v}\right\}$ to $v^{*}$}

Under 2.13)-(2.17), conclusion (c) of Theorem 2.1 shows that the increasing sequence $\left\{B^{n} \underline{v}\right\}_{n=1}^{\infty}$ converges to the value function $v^{*}$ pointwise. The natural question is, "Does the decreasing sequence $\left\{B^{n} \bar{v}\right\}_{n=1}^{\infty}$ also converge to $v^{*}$ ?" The answer is no in general.

To verify this, let $\alpha>0$ and suppose that $\alpha<\beta<1$. Consider the symbolic diagram depicted in Figure 3 , more precisely, assume the following:

$$
\begin{gathered}
X=\left\{(i, j): i, j \in \mathbb{Z}_{+}, j \leq i\right\}, \\
\Gamma((i, j))= \begin{cases}\left\{\left(i^{\prime}, 0\right): i^{\prime} \in \mathbb{Z}_{+}\right\} & \text {if }(i, j)=(0,0), \\
\{(i, j)\} & \text { if } i=j \neq 0, \\
\{(i, j+1)\} & \text { if } j<i,\end{cases} \\
u\left((i, j),\left(i^{\prime}, j^{\prime}\right)\right)= \begin{cases}-\alpha & \text { if }(i, j)=\left(i^{\prime}, j^{\prime}\right)=(0,0), \\
-\beta^{-i} & \text { if }(i, j)=\left(i^{\prime}, j^{\prime}\right) \neq(0,0), \\
0 & \text { otherwise. }\end{cases}
\end{gathered}
$$

The value function $v^{*}$ can be computed directly:

$$
v^{*}((i, j))= \begin{cases}-\alpha /(1-\beta) & \text { if }(i, j)=(0,0), \\ -\beta^{-j} /(1-\beta) & \text { otherwise. }\end{cases}
$$

Let $\underline{v}=v^{*}$ and $\bar{v}=0$. Then $\underline{v} \leq \bar{v}$ and $B \underline{v}=\underline{v}$. Since $u \leq 0$, we have $B \bar{v} \leq \bar{v}$. Thus (2.13)-(2.15) hold. As any feasible path eventually becomes constant, 2.16) and (2.17) hold with equality. Hence Theorem 2.1 applies.

\footnotetext{
${ }^{16}$ Let $i \in \mathbb{N}$. Then at state $(i, i)$, we have $v^{*}((i, i))=-\beta^{-i} /(1-\beta)$. Note that $v^{*}((i, i-$ $k))=\beta^{k} v^{*}((i, i))$ for $k=1, \ldots, i$; thus $v^{*}((i, j))=-\beta^{i-j} v^{*}((i, i))=-\beta^{-j} /(1-\beta)$. It remains to compute $v((0,0))$. If $x_{t}=(0,0)$ for all $t \in \mathbb{Z}_{+}$, then $S\left(\left\{x_{t}\right\}_{t=0}^{\infty}\right)=-\alpha /(1-\beta)$. If $x_{1}=(i, 0)$ with $i>0$, then $S\left(\left\{x_{t}\right\}_{t=0}^{\infty}\right)=\beta v^{*}((i, 0))=-\beta /(1-\beta)<-\alpha /(1-\beta)$. Hence it is never optimal to leave state $(0,0)$, so that $v^{*}((0,0))=-\alpha /(1-\beta)$.
} 


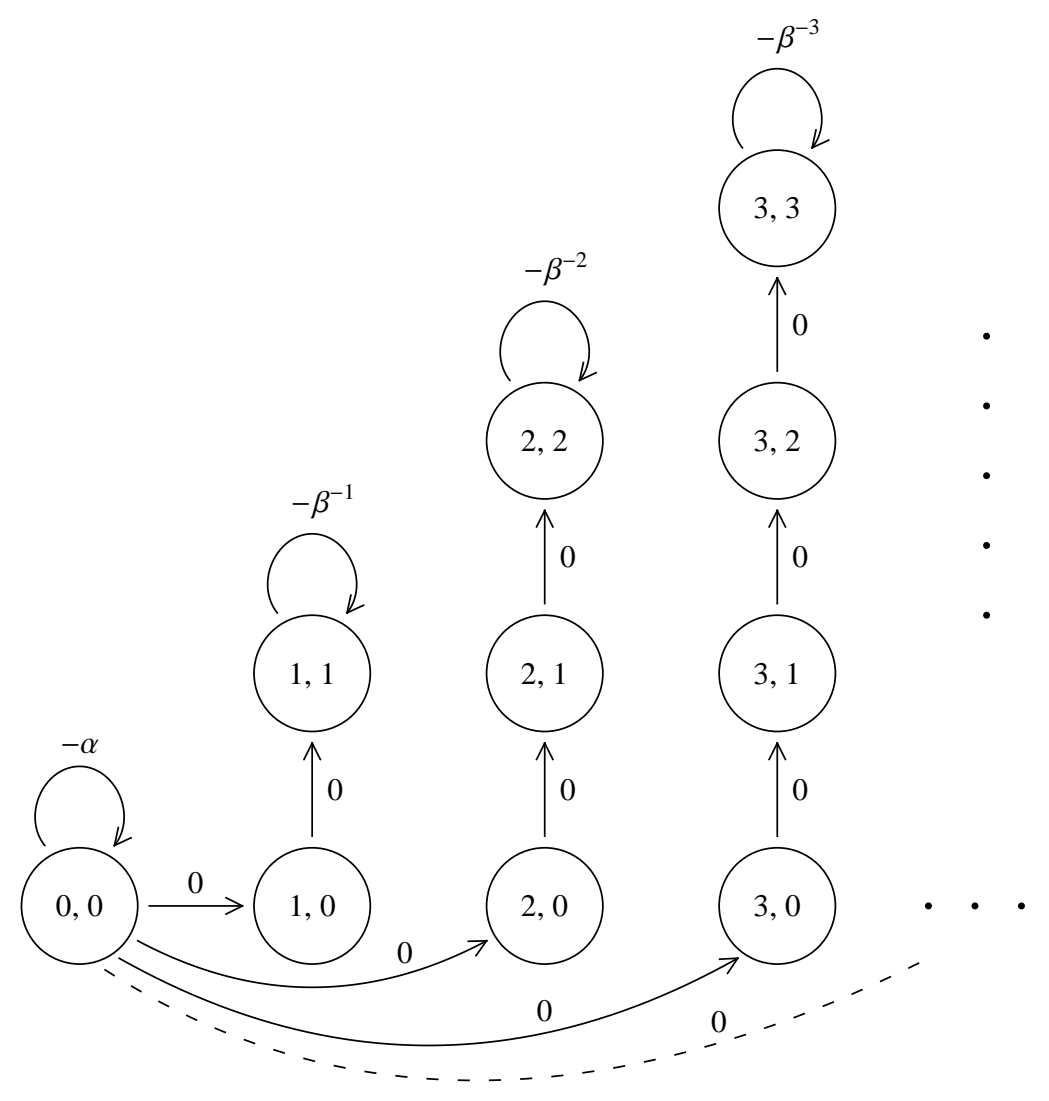

Figure 3: States $(i, j) \in X$ (circles), feasible transitions (arrows), and associated returns (values adjacent to arrows) under (4.9)-4.11) 
Consider the decreasing sequence $\left\{\bar{v}_{n}\right\}_{n=1}^{\infty} \equiv\left\{B^{n} \bar{v}\right\}_{n=1}^{\infty}$. If $(i, j) \neq(0,0)$, there is only one feasible transition from $(i, j)$, so that $\bar{v}_{n}((i, j))$ can be computed directly:

$$
\bar{v}_{n}((i, j))= \begin{cases}-\beta^{-j} \sum_{k=0}^{n-(i-j)-1} \beta^{k} & \text { if } i>0 \text { and } n \geq i-j+1, \\ 0 & \text { otherwise. }\end{cases}
$$

This formula works for $(i, j)=(0,0)$ as well; i.e., $\bar{v}_{n}((0,0))=0$ for all $n \in \mathbb{N} 17$

Now letting $\bar{v}^{*}=\lim _{n \uparrow \infty} \bar{v}_{n}$, we see that $\bar{v}^{*}((i, j))=v^{*}((i, j))$ for all $(i, j) \in X \backslash\{(0,0)\}$, but $\bar{v}^{*}((0,0))=0>v^{*}((0,0))$; i.e., the sequence $\left\{\bar{v}_{n}\right\}_{n=1}^{\infty}$ fails to converge to $v^{*}$ at $(0,0)$.

Interestingly, the sequence $\left\{B^{n} \bar{v}^{*}\right\}_{t=1}^{\infty}$ restarted from $\bar{v}^{*}$ converges to $v^{*}$. Indeed, $\left(B^{n} \bar{v}^{*}\right)((0,0))=-\alpha\left(1+\beta+\cdots+\beta^{n-1}\right) \rightarrow v^{*}((0,0))$ as $n \uparrow \infty$.

\section{Concluding Remarks}

In this paper we have established some elementary results on solutions to the Bellman equation - or fixed points of the Bellman operator-without introducing any topological assumption. Our main results are twofold. First, given an order interval of functions that is mapped into itself by the Bellman operator, if the upper and lower boundaries of the order interval satisfy transversality-like conditions, then (a) the Bellman equation has a unique solution in this order interval, (b) this solution is the value function, and (c) the value function can be computed by value iteration starting from the lower boundary of the order interval. Second, if there exists a function that is mapped upward by the Bellman operator, and if this function is dominated by the value function and satisfies a transversality-like condition, then the value function can be computed by value iteration starting from this function. We have derived various known results as consequences of these results to clarify how they relate to existing ones. We have applied our results to two optimal growth models, one with a discontinuous production function, the other with "roughly increasing" returns. We have also presented an example to show that even under the conditions of our first main result, value iteration

\footnotetext{
${ }^{17}$ To see this, define $\bar{v}_{0}=\bar{v}=0$. Then $\bar{v}_{0}((0,0))=0$. Let $n \in \mathbb{Z}_{+}$. With $\bar{v}_{n}$ given by 4.13), we have $\bar{v}_{n+1}((0,0))=\beta \sup _{i \in X} \bar{v}_{n}((i, 0))=0$ since $\bar{v}_{n}((i, 0))=0$ for all $i \geq n$. By induction, $\bar{v}_{n}((0,0))=0$ for all $n \in \mathbb{N}$.
} 
starting from the upper boundary of the order interval need not converge to the value function.

Our results are elementary in that they do not require any knowledge of mathematics beyond undergraduate analysis. Therefore, they are highly accessible to applied researchers as well as graduate students. Even in situations where standard topological assumptions hold, our results are considerably simpler than most of the existing results on fixed points of the Bellman operator. In addition, our results, which require no topological assumption, apply to models with discontinuities and even to those with increasing returns.

On the other hand, our results directly establish virtually no property of the value function. This is in sharp contrast to the existing results based on variants of the contraction mapping theorem. Regarding this matter, we have shown how the increasing, convergent sequence given by our results can be used to derive certain properties of the value function. We plan to pursue applications of this idea as well as extensions to stochastic models in future research.

\section{Appendix A Proofs}

\section{A.1 Proof of Theorem 2.1}

The proof consists of three lemmas and a concluding argument. The proof of the first lemma slightly generalizes an argument of Stokey and Lucas (1989, Theorem 4.3). The second lemma essentially shows that $B^{T} v$ with $T \in \mathbb{N}$ and $v \in V$ is the value function of the $T$ period problem with the value of the terminal stock $x_{T}$ given by $v\left(x_{T}\right)$. This result extends the classical idea of Bertsekas and Shreve (1978, Section 3.2) to our setting. The last lemma is less trivial than the first two. The concluding argument applies the Knaster-Tarski fixed point theorem and combines the first and last lemmas.

Lemma A.1. Let $\bar{v} \in V$ satisfy (2.17). Let $v \in V$ be a fixed point of $B$ with $v \leq \bar{v}$. Then $v \leq v^{*}$.

Proof. Let $x_{0} \in X$. If $v\left(x_{0}\right)=-\infty$, then $v\left(x_{0}\right) \leq v^{*}\left(x_{0}\right)$. Consider the case $v\left(x_{0}\right)>-\infty$. Let $\epsilon>0$. Let $\left\{\epsilon_{t}\right\}_{t=0}^{\infty} \subset(0, \infty)$ be such that $\sum_{t=0}^{\infty} \beta^{t} \epsilon_{t} \leq \epsilon$. Since $v=B v$, for any $t \in \mathbb{Z}_{+}$and $x_{t} \in X$, there exists $x_{t+1} \in \Gamma\left(x_{t}\right)$ such that

$$
v\left(x_{t}\right) \leq u\left(x_{t}, x_{t+1}\right)+\beta v\left(x_{t+1}\right)+\epsilon_{t} .
$$


We pick $x_{1} \in \Gamma\left(x_{0}\right), x_{2} \in \Gamma\left(x_{1}\right), \ldots$ so that A.1 holds for all $t \in \mathbb{Z}_{+}$. Then $\left\{x_{t}\right\}_{t=1}^{\infty} \in \Pi\left(x_{0}\right)$. By repeated application of (A.1) we have

$$
\begin{aligned}
v\left(x_{0}\right) \leq & u\left(x_{0}, x_{1}\right)+\beta v\left(x_{1}\right)+\epsilon_{0} \\
\leq & u\left(x_{0}, x_{1}\right)+\beta\left[u\left(x_{1}, x_{2}\right)+\beta v\left(x_{2}\right)+\epsilon_{1}\right]+\epsilon_{0} \\
& \vdots \\
\leq & \sum_{t=0}^{T-1} \beta^{t} u\left(x_{t}, x_{t+1}\right)+\beta^{T} v\left(x_{T}\right)+\epsilon, \quad \forall T \in \mathbb{N} .
\end{aligned}
$$

Since $v\left(x_{0}\right)>-\infty$, we have $\beta^{T} v\left(x_{T}\right)>-\infty$ for all $T \in \mathbb{N}$. It follows that

$$
v\left(x_{0}\right)-\epsilon-\beta^{T} v\left(x_{T}\right) \leq \sum_{t=0}^{T-1} \beta^{t} u\left(x_{t}, x_{t+1}\right) .
$$

Applying $\underline{\lim }_{T \uparrow \infty}$ to both sides and recalling 2.5$)$ and $(2.6)$, we have

$$
v\left(x_{0}\right)-\epsilon-\varlimsup_{T \uparrow \infty} \beta^{T} v\left(x_{T}\right) \leq \varliminf_{T \uparrow \infty} \sum_{t=0}^{T-1} \beta^{t} u\left(x_{t}, x_{t+1}\right) \leq S\left(\left\{x_{t}\right\}_{t=0}^{\infty}\right) \leq v^{*}\left(x_{0}\right) .
$$

By 2.17) we have $v\left(x_{0}\right)-\epsilon \leq v^{*}\left(x_{0}\right)$. Since this is true for any $\epsilon>0$, we have $v\left(x_{0}\right) \leq v^{*}\left(x_{0}\right)$. Since $x_{0}$ is arbitrary, we obtain $v \leq v^{*}$.

For any $v \in V$, define $v_{1}=B v$; for each $n \in \mathbb{N}$, provided that $v_{n} \in V$, define $v_{n+1}=B v_{n}$. The following remark follows from 2.11.

Remark A.1. Let $v, w \in V$ satisfy $v \leq w$ and $B w \leq w$. Then for all $n \in \mathbb{N}$, we have $v_{n} \leq w$ and thus $v_{n} \in V$.

Lemma A.2. Let $\bar{v} \in V$ satisfy 2.15). Let $v \in V$ satisfy $v \leq \bar{v}$. Then for any $T \in \mathbb{N}$, we have $v_{T} \in V$ and

$$
\forall x_{0} \in X, \quad v_{T}\left(x_{0}\right)=\sup _{\left\{x_{t}\right\}_{t=1}^{\infty} \in \Pi\left(x_{0}\right)}\left\{\sum_{t=0}^{T-1} \beta^{t} u\left(x_{t}, x_{t+1}\right)+\beta^{T} v\left(x_{T}\right)\right\} .
$$

Proof. Note from (2.15) and Remark A.1 with $w=\bar{v}$ that $v_{n} \in V$ for all $n \in \mathbb{N}$. For any $x_{0} \in X$, we have

$$
v_{1}\left(x_{0}\right)=\sup _{x_{1} \in \Gamma\left(x_{0}\right)}\left\{u\left(x_{0}, x_{1}\right)+\beta v\left(x_{1}\right)\right\}
$$




$$
\begin{aligned}
& =\sup _{x_{1} \in \Gamma\left(x_{0}\right)} \sup _{\left\{x_{t}\right\}_{t=2}^{\infty} \in \Pi\left(x_{1}\right)}\left\{u\left(x_{0}, x_{1}\right)+\beta v\left(x_{1}\right)\right\} \\
& =\sup _{\left\{x_{t}\right\}_{t=1}^{\infty} \in \Pi\left(x_{0}\right)}\left\{u\left(x_{0}, x_{1}\right)+\beta v\left(x_{1}\right)\right\}
\end{aligned}
$$

where A.10 holds because $\left\{u\left(x_{0}, x_{1}\right)+\beta v\left(x_{1}\right)\right\}$ is independent of $\left\{x_{t}\right\}_{t=2}^{\infty}{ }^{18}$ and A.11 follows by combining the two suprema (see Kamihigashi, 2008, Lemma 1). It follows that A.8 holds for $T=1$.

Now assume A.8 for $T=n \in \mathbb{N}$. For any $x_{0} \in X$, we have

$$
\begin{aligned}
v_{n+1}\left(x_{0}\right)= & \sup _{x_{1} \in \Gamma\left(x_{0}\right)}\left\{u\left(x_{0}, x_{1}\right)+\beta v_{n}\left(x_{1}\right)\right\} \\
= & \sup _{x_{1} \in \Gamma\left(x_{0}\right)}\left\{u\left(x_{0}, x_{1}\right)\right. \\
& \left.\quad+\beta \sup _{\left\{x_{i+1}\right\}_{i=1}^{\infty} \in \Pi\left(x_{1}\right)}\left\{\sum_{i=0}^{n-1} \beta^{i} u\left(x_{i+1}, x_{i+2}\right)+\beta^{n} v\left(x_{n+1}\right)\right\}\right\} \\
= & \sup _{x_{1} \in \Gamma\left(x_{0}\right)}\left\{\sup _{i+1}\right\}_{i=1}^{\infty} \in \Pi\left(x_{1}\right) \\
& \left.\sum_{\sup _{t=0}}^{n} \beta^{t} u\left(x_{t}, x_{t+1}\right)+\beta^{n+1} v\left(x_{n+1}\right)\right\} \\
= & \left\{\sum_{\left\{x_{t}\right\}_{t=1}^{\infty} \in \Pi\left(x_{0}\right)}^{n} \beta^{t} u\left(x_{t}, x_{t+1}\right)+\beta^{n+1} v\left(x_{n+1}\right)\right\}
\end{aligned}
$$

where A.13 uses A.8 for $T=n$, A.14 holds because $u\left(x_{0}, x_{1}\right)$ is independent of $\left\{x_{i+1}\right\}_{i=1}^{\infty}$, and A.15 follows by combining the two suprema (see Kamihigashi, 2008, Lemma 1). It follows that A.8 holds for $T=n+1$. By induction, A.8 holds for all $T \in \mathbb{N}$.

Lemma A.3. Let $\underline{v}, \bar{v} \in V$ satisfy (2.13)-(2.16). Then $\underline{v}^{*} \equiv \lim _{T \uparrow \infty} \underline{v}_{T} \geq$ $v^{*}, 19$

Proof. Note from 2.13 $2.15,2.11$, and Remark A.1 that $\left\{\underline{v}_{T}\right\}_{T=1}^{\infty}$ is an increasing sequence in $V$. Thus for any $x_{0} \in X$, we have

$$
\begin{aligned}
\underline{v}^{*}\left(x_{0}\right) & =\sup _{T \in \mathbb{N}} \underline{v}_{T}\left(x_{0}\right) \\
& =\sup _{T \in \mathbb{N}} \sup _{\left\{x_{t}\right\}_{t=1}^{\infty} \in \Pi\left(x_{0}\right)}\left\{\sum_{t=0}^{T-1} \beta^{t} u\left(x_{t}, x_{t+1}\right)+\beta^{T} \underline{v}\left(x_{T}\right)\right\}
\end{aligned}
$$

\footnotetext{
${ }^{18}$ This step uses the assumption that $\Gamma$ is nonempty-valued.

${ }^{19}$ Here $\underline{v}_{T}=B^{T} \underline{v}$ for all $T \in \mathbb{N}$ and $\underline{v}^{*}(x)=\lim _{T \uparrow \infty} \underline{v}_{T}(x)$ for all $x \in X$.
} 


$$
\begin{aligned}
& =\sup _{\left\{x_{t}\right\}_{t=1}^{\infty} \in \Pi\left(x_{0}\right)} \sup _{T \in \mathbb{N}}\left\{\sum_{t=0}^{T-1} \beta^{t} u\left(x_{t}, x_{t+1}\right)+\beta^{T} \underline{v}\left(x_{T}\right)\right\} \\
& \geq \sup _{\left\{x_{t}\right\}_{t=1}^{\infty} \in \Pi^{0}\left(x_{0}\right)} \operatorname{L}_{T \uparrow \infty}\left\{\sum_{t=0}^{T-1} \beta^{t} u\left(x_{t}, x_{t+1}\right)+\beta^{T} \underline{v}\left(x_{T}\right)\right\} \\
& \geq \sup _{\left\{x_{t}\right\}_{t=1}^{\infty} \in \Pi^{0}\left(x_{0}\right)}\left\{\underset{T \uparrow \infty}{\mathrm{L}} \sum_{t=0}^{T-1} \beta^{t} u\left(x_{t}, x_{t+1}\right)+\underline{\lim }_{T \uparrow \infty} \beta^{T} \underline{v}\left(x_{T}\right)\right\} \\
& \geq \sup _{\left\{x_{t}\right\}_{t=1}^{\infty} \in \Pi^{0}\left(x_{0}\right)} \underset{T \uparrow \infty}{\mathrm{L}} \sum_{t=0}^{T-1} \beta^{t} u\left(x_{t}, x_{t+1}\right)=v^{*}\left(x_{0}\right),
\end{aligned}
$$

where A.17) uses Lemma A.2 (A.18) follows by interchanging the two suprema (see Kamihigashi, 2008, Lemma 1), A.19 holds because $\Pi^{0}\left(x_{0}\right) \subset$ $\Pi\left(x_{0}\right)$ (recall (2.8)) and $\mathrm{L}_{T \uparrow \infty} a_{T} \leq \sup _{T \in \mathbb{N}} a_{T}$ for any sequence $\left\{a_{T}\right\}$ in

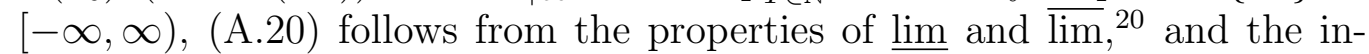
equality in (A.21) uses 2.16). It follows that $\underline{v}^{*} \geq v^{*}$.

To complete the proof of Theorem 2.1, suppose that there exist $\underline{v}, \bar{v} \in V$ satisfying (2.13)-(2.17). The order interval $[\underline{v}, \bar{v}]$ is partially ordered by $\leq$ (recall (2.10)). Given any $F \subset[\underline{v}, \bar{v}]$, we have $\sup F \in[\underline{v}, \bar{v}]$ because

$$
\forall x \in X, \quad(\sup F)(x)=\sup \{f(x): f \in F\} \in[\underline{v}(x), \bar{v}(x)] .
$$

Since $B$ is a monotone operator, and since $B([\underline{v}, \bar{v}]) \subset[\underline{v}, \bar{v}]$ by $2.13-2.15$ and (2.11), it follows that $B$ has a fixed point $v$ in $[\underline{v}, \bar{v}]$ by the KnasterTarski fixed point theorem (e.g., Aliprantis and Boder, 2006, p. 16). Since $\underline{v} \leq v=B v$, we have $\underline{v}_{n} \leq v$ for all $n \in \mathbb{N}$ by Remark A.1 thus $\underline{v}^{*} \leq v{ }^{21}$ Since $v \leq v^{*}$ by Lemma A.1, and since $v^{*} \leq v^{*}$ by Lemma A.3, it follows that $v \leq v^{*} \leq \underline{v}^{*} \leq v$. Hence $v=\underline{v}^{*}=v^{*}$. Therefore $v^{*}$ is a unique fixed point of $B$ in $[\underline{v}, \bar{v}]$; this establishes (a) and (b). Finally (c) holds because $\underline{v}^{*}=v^{*}$.

\section{A.2 Proof of Proposition 2.1}

Let $\bar{v} \in V$ satisfy (2.17) and (2.18). Then by Lemma A.1, any fixed point $v$ of $B$ with $v \leq \bar{v}$ satisfies $v \leq v^{*}$. Since $v^{*} \leq \bar{v}$ and $v^{*}$ is a fixed point of $B$

\footnotetext{
${ }^{20}$ We have $\underline{\lim }\left(a_{t}+b_{t}\right) \geq \underline{\lim } a_{t}+\underline{\lim } b_{t}$ and $\varlimsup \lim \left(a_{t}+b_{t}\right) \geq \varlimsup \lim a_{t}+\underline{\lim } b_{t}$ for any sequences $\left\{a_{t}\right\}$ and $\left\{b_{t}\right\}$ in $[-\infty, \infty)$ whenever both sides are well-defined (e.g., Michel, 1990, p. 706).

${ }^{21}$ See footnote 19 for the definitions of $\underline{v}_{n}$ and $\underline{v}^{*}$.
} 
by Lemma 2.1, it follows that $v^{*}$ is the largest fixed point of $B$ in $[-\infty, \bar{v}]$.

\section{A.3 Proof of Therem 2.2}

Let $\underline{v} \in V$ satisfy (2.14), (2.16), and (2.19). Since $B v^{*}=v^{*}$ by Lemma 2.1. (2.13) -2.15 hold with $\bar{v}=v^{*}$. Hence by Lemma A.3, $\underline{v}^{*} \geq v^{*}$. Since $\underline{v} \leq v^{*}$, we also have $\underline{v}^{*} \leq v^{*}$. Thus $\underline{v}^{*}=v^{*}$. We have verified the second conclusion. If $B$ has a fixed point $v \in[\underline{v}, \infty]$, then $v^{*}=\underline{v}^{*} \leq v$; thus the first conclusion also holds.

\section{References}

Aliprantis, C.D., Border, K.C., 2006, Infinite Dimensional Analysis: A Hitchhiker's Guide, 3rd Edition, Springer-Verlag, Berlin.

Algan, Y., Challe, E., Ragot, X., 2011, Incomplete markets and the outputinflation tradeoff, Economic Theory 46, 55-84.

Alvarez, F., Stokey, N.L., 1998, Dynamic programming with homogenous functions, Journal of Economic Theory 82, 167-189.

Azariadis, C., Drazen, A., 1990, Threshold externalities in economic development," Quarterly Journal of Economics 105, 501-26.

Amir, R., Mirman, L.J., Perkins, W.R., 1991, One-sector nonclassical optimal growth: optimality conditions and comparative dynamics, International Economic Review 32, 625-644.

Bertsekas, D.P., Shreve, S.E., 1978, Stochastic Optimal Control: The Discrete Time Case, Academic Press, New York.

Bloch, F., Houy, N., 2012, Optimal assignment of durable objects to successive agents, Economic Theory 51, 13-33.

Durán, J, 2000, On dynamic programming with unbounded returns, Economic Theory 15, 339-352.

Dutta, P.K., Mitra, T., 1989a, On continuity of the utility function in intertemporal allocation models: an example, International Economic Review $30,527-536$.

Dutta, P.K., Mitra, T., 1989b, Maximum theorems for convex structures with an application to the theory of optimal intertemporal allocation, Journal of Mathematical Economics 18, 77-86. 
Dutta, P.K., Radner, R., 2012, Capital growth in a global warming model: will China and India sign a climate treaty? Economic Theory 49, 411443.

Goenka, A., Lin, L., 2012, Infectious diseases and endogenous fluctuations, Economic Theory 50, 125-149.

Herrera, H., Martinelli, C., 2013, Oligarchy, democracy, and state capacity, Economic Theory 52, 165-186.

Kamihigashi, T., 2008, On the principle of optimality for nonstationary deterministic dynamic programming, International Journal of Economic Theory 4, 519-525.

Kamihigashi, T., Roy, S., 2006, Dynamic optimization with a nonsmooth, nonconvex technology: the case of a linear objective function, Economic Theory 29, 325-340.

Kamihigashi, T., Roy, S., 2007, A nonsmooth, nonconvex model of optimal growth, Journal of Economic Theory 132, 435-460.

Kamihigashi, T., Stachurski, J., 2011, Existence, stability and computation of stationary distributions: an extension of the Hopenhayn-Prescott theorem, RIEB Discussion Paper No. 2011-32, RIEB, Kobe University.

Kamihigashi, T., 2013, An order-theoretic approach to dynamic programming: an exposition, Economic Theory Bulletin, forthcoming.

Kamihigashi, T., Furusawa, T., 2010, Global dynamics in repeated games with additively separable payoffs, Review of Economic Dynamics 13, 899-918.

Karp, L., Zhang, J., 2012, Tases versus quantities for a stock pollutant with endogenous abatement costs and asymmetric information, Economic Theory $49,371-409$.

Le Van, C., Morhaim, L, 2002, Optimal growth models with bounded or unbounded returns: a unifying approach, Journal of Economic Theory 105, $158-187$.

Llanes, G., Trento, S., 2012, Paten policy, patent pools, and the accumulation of claims in sequential innovation, Economic Theory 50, 703-725.

Martins-da-Rocha, V.F., Vailakis, Y., 2010, Existence and uniqueness of a fixed point for local contractions, Econometrica 78, 1127-1141.

Matkowski, J., Nowak, A.S., 2011, On discounted dynamic programming with unbounded returns, Economic Theory 46, 455-474. 
Michel, P., 1990, Some clarifications on the transversality condition, Econometrica 58, 705-723.

Muradian, R., 2001, Ecological thresholds: a survey, Ecological Economics $38,7-24$.

Nævdal, R., 2003, Optimal regulation of natural resources in the presence of irreversible threshold effects, Natural Resource Modeling 16, 305-333.

Reis, C., 2013, Taxation without commitment, Economic Theory 52, 565588 .

Rinćon-Zapatero, J.P., Rodríguez-Palmero, C., 2003, Existence and uniqueness of solutions to the Bellman equation in the unbounded case, Econometrica 71, 1519-1555.

Rinćon-Zapatero, J.P., Rodríguez-Palmero, C., 2007, Recursive utility with unbounded aggregators, Economic Theory 33, 381-391.

Rinćon-Zapatero, J.P., Rodríguez-Palmero, C., 2009, Corrigendum to "Existence and uniqueness of solutions to the Bellman equation in the unbounded case" Econometrica, Vol. 71, No. 5 (September, 2003), 15191555, Econometrica 77, 317-318.

Romer, P., 1986, Increasing returns and long-run growth, Journal of Political Economy 94, 1002-1037.

Roy, S., Zilcha, I., 2012, Stochastic growth with short-run prediction of shocks, Economic Theory 51, 539-580.

Schaar, M.v.d., Xu, J., Zame, W., 2013, Efficient online exchange via fiat money, Economic Theory 54, 211-248.

Stokey, N., Lucas, R.E., Jr., 1989, Recursive Methods in Economic Dynamics, Harvard University Press, Cambridge, MA.

Strauch, R.E., 1966, Negative dynamic programming, Annals of Mathematical Statistics 37, 871-890. 